\title{
Orientation-specific possibility priming for novel three-dimensional objects
}

\author{
PEPPER WILLIAMS \\ University of Massachusetts, Boston, Massachusetts \\ and \\ MICHAEL J. TARR \\ Brown University, Providence, Rhode Island
}

\begin{abstract}
Priming effects on the object possibility task, in which participants decide whether line drawings could or could not be possible three-dimensional objects, may be supported by the same processes and representations used in recognizing and identifying objects. Three experiments manipulating objects' picture-plane orientation provided limited support for this hypothesis. Like old/new recognition performance, possibility priming declined as study-test orientation differences increased from $0^{\circ}$ to $60^{\circ}$. However, while significant possibility priming was not observed for larger orientation differences, recognition performance continued to decline following $60^{\circ}-180^{\circ}$ orientation shifts. These results suggest that possibility priming and old/new recognition may rely on common viewpoint-specific representations but that access to these representations in the possibility test occurs only when study and test views are sufficiently similar (i.e., rotated less than $60^{\circ}$ ).
\end{abstract}

The object possibility paradigm was developed by Schacter, Cooper, and their colleagues (Schacter, Cooper, \& Delaney, 1990) to study implicit memory for novel three-dimensional (3-D) objects. The test task is to judge whether line drawings represent possible objects that could potentially exist in three dimensions or impossible objects that could not actually exist in the real 3-D world (Figure 1). As in other implicit memory paradigms, participants first study a series of stimuli and then are tested on studied and unstudied items. Implicit memory is revealed if participants show significant performance differences for studied test items compared with unstudied test items, and such a performance difference is known as a priming effect.

In addition to the object possibility test, many of Schacter and Cooper's experiments have included an old/new recognition test. The latter is an explicit memory test, because participants must explicitly determine whether test items were or were not included in the study list. Most of Schacter and Cooper's studies have been designed to show that old/new recognition performance can be mediated by

This research was supported by Air Force Office of Scientific Research Grant F49620-91-J0169 to M.J.T. and a TRANSCOOP grant to M.J.T. and Heinrich Bülthoff. Experiments 1 and 2 were first reported in a poster given at the 37th Annual Meeting of the Psychonomic Society, Chicago, November 1-3, 1996. Thanks to Jim Tanaka and Tim Curran for facilitating data collection at Oberlin College and Case Western Reserve University, respectively. Copies of the stimuli used in the experiments are available on the World-Wide Web at http://psych.umb.edu/ williams/stimuli/. Correspondence should be addressed to P. Williams, Department of Psychology, University of Massachusetts, 100 Morrissey Blvd., Boston, MA 02125 (e-mail: pepper.williams@umb.edu).

-Accepted by previous editor, Myron L. Braunstein different sources of encoded information from the source mediating object possibility priming. More specifically, Cooper and Schacter (1992, p. 145) argued that information regarding "the global, three-dimensional organization of parts" of objects is responsible for object possibility priming and is encoded separately from information regarding objects' identity, function, and other "meaningful" aspects, which is used in making old/new recognition decisions. From a cognitive neuropsychological perspective (Schacter, 1992), determining the neuroanatomical loci of encoded information is a high priority, and the efforts of Schacter, Cooper, and their colleagues have proven fruitful in this regard (Schacter et al., 1995).

The object possibility task may also be useful in the study of the perceptual processes involved in object recognition. Many object recognition researchers prefer to use novel objects as stimuli, in order to gain precise control over the conditions under which objects are learned (Tarr \& Pinker, 1989). However, such a strategy places limitations on the test tasks that can be used in assessing participants' object recognition performance. The predominant task in object recognition research with familiar objects has been naming (i.e., a picture of an elephant is shown to the participant, and the participant says "elephant," e.g., Jolicoeur, 1985); however, to use a naming task with novel stimuli, participants must first be trained on the names of the stimuli. This training is time-consuming, and it limits the number of stimuli that can be used in an experiment (since it is difficult for participants to learn more than $7 \pm 2$ object names). In lieu of naming, other researchers (e.g., Rock, Di Vita, \& Barbeito, 1981) have employed the same old/new recognition task used by Schacter, Cooper, and colleagues (although old/new recognition can be influenced by nonvisual factors such as 

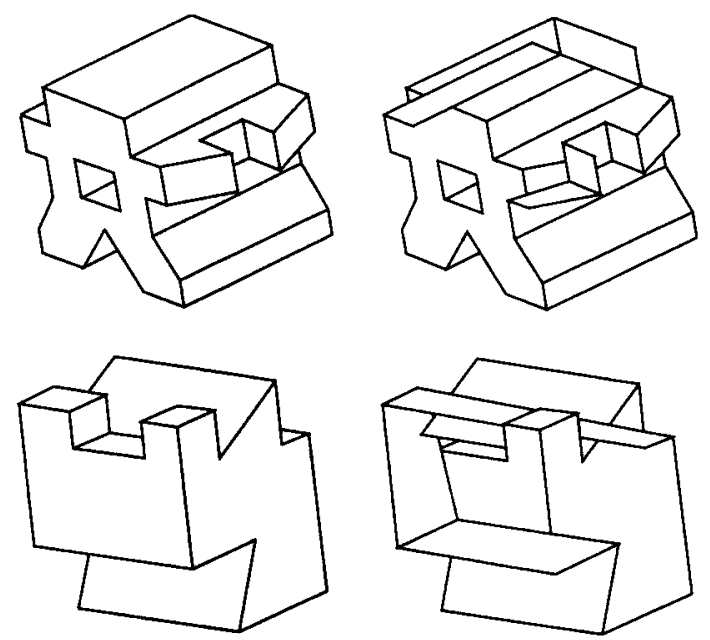

Figure 1. Possible (left) and impossible (right) versions of two objects. Possible figures could represent real, 3-D objects; impossible figures contain structural anomalies, rendering them unable to be instantiated in three dimensions.

semantic associations, it also undoubtably requires an act of object recognition). However, most of our interactions with objects probably do not involve an explicit act of memory retrieval, so this task may not be ideal, either.

All this is not to say that experiments using naming or old/new recognition should be discounted -object recognition occurs in many experimental and environmental contexts, so the best strategy is to seek converging evidence by employing as many different tasks as possible. The object possibility task may be a valuable addition to the object recognition researcher's bag of tricks, because encoded representations of objects are only implicitly accessed during the task, but participants need not learn names for the novel stimuli. Our goal in the present study was to connect the object possibility paradigm with the object recognition literature by exploring the effects on possibility priming of a variable, picture-plane orientation, that has been studied intensively by object recognition researchers (for a review, see Tarr \& Pinker, 1989). In the three experiments reported here, objects were studied and tested either in the same or in different pictureplane orientations (Figures 2, 4, and 7 show the studied and tested orientations used in the experiments). Both object possibility and old/new recognition tasks were employed in order to compare orientation effects on priming with orientation effects on explicit object identification.

Cooper, Schacter, and Moore (1991) reported two experiments of this sort, in which studied objects were tested in the same orientation as studied, rotated $180^{\circ}$ (Experiment 1), or rotated $\pm 120^{\circ}$ (Experiment 2). Priming effects in the rotated conditions of these experiments were not significant, so Cooper et al. concluded that possibility priming is an all-or-none phenomenon with respect to orientation shifts. Numerically, however, the mean priming effect for unrotated objects was largest, followed by $120^{\circ}$-rotated objects and then $180^{\circ}$-rotated objects, sug- gesting that a trend of decreasing priming with increasing orientation shifts might be obtainable with more orientation conditions and/or a within-participants design.

In the present experiments, study-to-test orientation shifts were manipulated more systematically than in Cooper et al.'s (1991) study, in an attempt to more precisely explicate the effects of object rotation on the structural processing that presumably underlies possibility priming. In Experiment 1, we replicated Cooper et al.'s finding that large orientation shifts of any magnitude drastically diminish possibility priming. However, in Experiments 2 and 3, we found that smaller orientation shifts led to more systematic effects on object possibility performance, allowing us to form new hypotheses about the processes and representations underlying performance on this and other object recognition tasks.

\section{EXPERIMENT 1}

In the study phase of Experiment 1, the participants viewed 24 possible objects in a predetermined canonical (or $0^{\circ}$ ) orientation, viewing each object four times while performing different study tasks. In the immediately following object possibility phase, the participants viewed the 24 studied objects and 12 unstudied objects in both their possible and their impossible versions (Figure 1). Both studied and unstudied test stimuli were divided into six orientation conditions, in which objects were rotated $0^{\circ}, \pm 60^{\circ}, \pm 120^{\circ}$, or $180^{\circ}$ relative to the studied orientation (Figure 2). The object possibility test was immediately

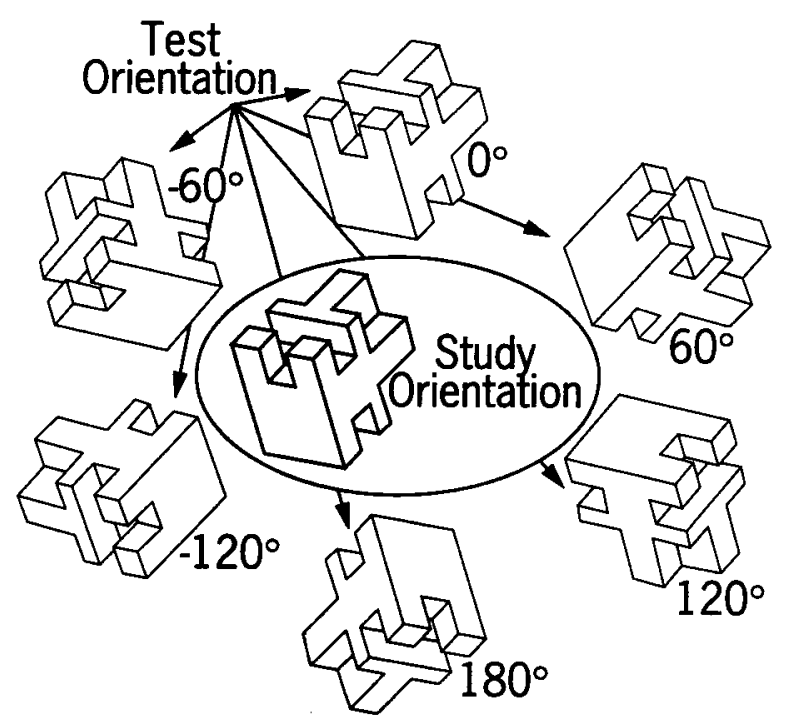

Figure 2. Stimulus design for Experiment 1. Objects were studied in an orientation that was rated by pilot participants to be highly "canonical" and then were tested in either the same orientation $\left(0^{\circ}\right.$ condition) or rotated $\pm 60^{\circ}, \pm 120^{\circ}$, or $180^{\circ}$ in the picture plane. In all experiments in this study, the participants saw only possible objects during study and old/new recognition tasks, but they saw both possible and impossible objects (see Figure 1) during the object possibility task. 
followed by an old/new recognition test, in which the participants viewed the 36 possible objects in the same orientations as in the object possibility phase.

Object possibility test figures were displayed for $5 \mathrm{sec}$ or until the participants responded, and response time (RT) served as the primary dependent measure (the participants were expected to be faster to respond to studied than to unstudied stimuli; Williams \& Tarr, 1997). As a continuous measure, we feel that RT is more suitable than accuracy for detecting effects of continuously varying independent measures, such as orientation. That is, the time taken to respond to a larger-orientation-shifted object can be expected to be longer than the time to respond to a smaller-orientation-shifted object. In contrast, when accuracy is the dependent measure (as in object possibility experiments from other laboratories; Ratcliff \& McKoon, 1995; Schacter et al., 1990), we gain only a limited amount of information about how well a participant performs on any one trial: All we can know is whether he/she did or did not receive enough information to make an accurate decision. Of course, by averaging over a large number of trials, we can convert these discrete observations into continuously varying accuracy rates; however, in the present experiments, relatively few observations were collectible for each study condition, so accuracy rates would still be a relatively coarse indicator of performance.

Two other methodological concerns, relevant to all three experiments, should be addressed here. First, as in any implicit memory study, care must be taken to avoid "contamination" of the implicit memory test by explicit memory. In other implicit tests, such as word fragment completion, for example, explicit memory of a study episode (e.g., remembering that the word ELEPHANT was recently seen) can help in performing the test task (completing the fragment_LEP___T). Our test procedure was specifically designed to avoid this pitfall, in that both possible and impossible versions of each object (Figure 1) were shown at test, and the participants were informed of this fact during practice trials. Thus, the fact that a test object seemed familiar (because it was studied) was not a valid indicator that the object was possible or impossible. Ratcliff and McKoon (1995, Experiment 6) showed that this technique can in fact eliminate effects of explicit memory on the object possibility test.

The second concern is that our within-participants manipulation of test task might somehow skew our old/new recognition results (note that since the object possibility task always preceded old/new recognition, the design could have no effect on the implicit task). Since the participants had already seen unstudied items in the object possibility task, our recognition test was more of a "source discrimination" task than a pure old/new task. Also, rotated studied objects had been seen in their new orientations during object possibility testing before the participants saw them in the recognition task. However, objects were seen four times for several seconds each in study tasks, and we hoped that this extensive contact during study would outweigh the few seconds of exposure to unstudied objects and rotated studied objects during object possibility. As it turned out, our old/new recognition results were quite consistent with past studies employing this task (e.g., Bülthoff \& Edelman, 1992), so any effect of the object possibility task on old/new recognition performance would seem to have been negligable.

\section{Method}

Participants. Data were analyzed for 36 students from the introductory psychology pool at Yale University ( 3 of which were replacements for participants who failed to follow task instructions or for whom experimenter error precluded analysis of their data).

Materials. The 36 objects were taken from those used in Williams and Tarr's (1997) study (see that paper for details of how the figures were created). Six copies of both the possible and the impossible versions of each object were rotated $0^{\circ}, \pm 60^{\circ}, \pm 120^{\circ}$, or $180^{\circ}$ in the picture plane (see Figure 2). An independent sample of 22 participants were shown all six rotations of each possible object and asked to decide which one of each group they thought was in the most "canonical" position. Agreement was fairly high among these judges; when there was disagreement, one of us (P.W.) arbitrarily picked one of the orientations to be the canonical view. Objects were always studied in this canonical viewpoint, which from here on will be referred to as the $0^{\circ}$ orientation. Stimuli were antialiased (resulting in smooth, grayscale lines) and measured approximately $7.5 \times$ $7.5 \mathrm{~cm}$ when shown on the Macintosh computer monitor. The participants viewed the figures from approximately $50 \mathrm{~cm}$ in front of the monitor, resulting in a visual angle of approximately $8.6^{\circ}$.

Procedure. All instructions were presented automatically to the participants on the computer monitor. They were initially told that the experiment would involve "observing line drawings of novel objects on the computer monitor and making some judgments about them." In the study phase, the participants viewed 24 objects, always in their possible versions and always in the $0^{\circ}$ orientation. They saw each object four times, each time in the context of a different encoding task: first, deciding what direction each object faced (choosing from "up-left," "straight-up," "up-right," etc.); second, deciding whether each object looked most like a building, a tool, or a spaceship; third, counting the number of visible surfaces in the figure; and fourth, rating how "aesthetically pleasing" each object seemed, on a 1-5 scale. Each object was shown for $5 \mathrm{sec}$ in each encoding task.

Immediately following the study tasks, the participants were given instructions for the object possibility task. They read that some figures in this task would "represent valid, possible 3-D objects that COULD exist in the real world," whereas other figures would "represent impossible objects that COULD NOT actually exist in the real 3-D world." The participants were told that the figures would stay on the monitor for up to $5 \mathrm{sec}$ each but that they should respond as quickly and as accurately as they could. The participants were also made aware of the fact that possible and impossible objects often looked quite similar to each other, so they had to examine each object carefully to make accurate decisions. Before testing, the participants performed 10 practice trials, in which they made a decision, were given feedback in the form of a "beep" if they chose incorrectly, and then saw the object again with a note explaining whether it was possible or impossible and, if the latter, highlighting the impossible portions of the figure. Following the practice trials, the participants were asked to inform the experimenter if they were not absolutely confident that they understood the distinction between possible and impossible objects. No more than 1 or 2 participants expressed any misgivings; 
when this happened, the experimenter showed them a picture of the practice objects and explained the distinction in greater detail.

Trials were computer-paced. Each began with a 1.5 -sec blank screen, followed by a $500-\mathrm{msec}$ fixation cross. The stimulus then appeared and stayed on the monitor until the participant responded or until $5 \mathrm{sec}$ had elapsed, whichever came first. The participants pressed the " $Z$ " key if they thought the figure was possible or the "M" key if they thought it was impossible. The sequence for the next trial then began. No feedback was given on test trials. There were 72 critical test trials, 36 objects ( 24 studied and 12 unstudied) that were each shown once in their possible versions and once in their impossible versions (Figure 1). Six objects (four studied and two unstudied) appeared at each of the six test orientations (Figure 2). The test also included eight "filler" trials, in which a possible object or an impossible object was shown twice in the same version. The practice trials also included a possible figure and an impossible figure, which were shown twice. These precautions were taken to ensure that the participants could not assume that once they had seen a possible version of an object, the next similar object would be an impossible version (or vice versa).

Immediately following the object possibility task, the participants received instructions for the old/new recognition test. Here, they were told that their task was to determine whether they had seen each object during one or more of the first four tasks they completed (the encoding tasks). It was made clear that the objects might appear in a different orientation than when they first saw them and that, if this was the case, they should still say that they had seen it before. The participants were also told that two thirds of the test objects were seen during study and that the other one third, to which they were to respond "new," were not seen on the study tasks but may have been seen during the object possibility task. Once they understood the instructions, the participants began the task immediately (there were no practice trials). As in the object possibility task, the sequence of events for each trial was 1.5-sec blank screen, then a 500-msec, fixation cross, then the stimulus for $5 \mathrm{sec}$ or until the participant responded. The participants pressed the " $Z$ " key to respond "old" (studied) or the " $M$ " key to respond "new" (unstudied). Following two filler trials (to get the participants used to pressing the correct keys), the participants viewed the same 36 possible objects in the same orientations as in the object possibility task.

Design and Analyses. Independent variables of interest were test version (possible or impossible), studied status (studied or unstudied), and test orientation, all of which were manipulated within participants. In an elaborate counterbalancing scheme, each object was placed in one of three "study groups" and one of six "orientation groups," and these were crossed to yield 18 stimulus sets. For example, in Stimulus Set 1, Study Groups 1 and 2 were studied, Study Group 3 was unstudied, Orientation Group 1 was tested in $0^{\circ}$ orientations, Orientation Group 2 was tested in $60^{\circ}$ orientations, and so on (all objects were shown in their $0^{\circ}$ orientations during study). Analyses included data from 2 participants in each stimulus set condition. Since there was no theoretical reason to suspect different patterns of results for clockwise and counterclockwise orientation shifts, results were collapsed over the direction of rotation in all experiments. Geometric means of correct RTs in each condition were calculated for each participant and served as the basis for analysis here and in the other two experiments (the use of geometric means reduces the effects of outlier response times; figures plot the arithmetic means of the participants' geometric means). Although we will only report results averaged across participants, items analyses were also conducted and resulted in similar $p$ values in all cases. An $\alpha$ level of .05 was adopted for all inferential statistics; actual significance levels are reported in special cases only.

\section{Results and Discussion}

Since objects were tested in both their possible and impossible versions, there was a potential for order ef- fects on the object possibility test. That is, the first presentation of an object in its impossible form could potentially prime the object's second presentation in its possible form, or vice versa. However, as in previous experiments (Williams \& Tarr, 1997), test order (first or second) did not significantly interact with variables of interest for either RT or accuracy in any of the experiments reported in this study, so all results were pooled over this factor.

An analysis of variance (ANOVA) on unstudied object possibility test items revealed no significant main or interaction effects involving test orientation on either accuracy or RT (all $F_{\mathrm{S}}<1.26$; mean accuracy rates were .97, $.94, .93$, and .94 , and mean RTs were $1,352,1,389,1,364$, and $1,345 \mathrm{msec}$, for unstudied possible objects in the $0^{\circ}$, $60^{\circ}, 120^{\circ}$, and $180^{\circ}$ viewpoints, respectively), so these data were pooled in subsequent analyses. Reported analyses thus included a test condition variable with five levels: studied and rotated $0^{\circ}$, studied and rotated $60^{\circ}$, studied and rotated $120^{\circ}$, studied and rotated $180^{\circ}$, and unstudied. As in previous experiments from our lab utilizing RT as the main dependent measure (Williams, 1995; Williams \& Tarr, 1997), there were no significant effects of test condition for impossible objects [accuracy, $F<1$; RT, $F(4,140)=2.16, M S_{\mathrm{e}}=81,297$; mean RTs were $1,386,1,454,1,466,1,564$, and $1,401 \mathrm{msec}$ in the $0^{\circ}, 60^{\circ}, 120^{\circ}, 180^{\circ}$, and unstudied test conditions]. Therefore, these trials were not analyzed further (see Williams \& Tarr, 1997, for discussion of the lack of RT priming for impossible test figures). A final preliminary analysis revealed no significant effect of test condition on accuracy rates for possible figures $\left[F(4,140)=1.96, M S_{\mathrm{e}}=0.011\right]$. This finding is again in agreement with previous ones from our lab (e.g., Williams \& Tarr, 1997), and it indicates that, given ample time to perform possibility decisions, the participants reached ceiling levels of accuracy.

The main focus of our object possibility analyses was the effect of test condition on RTs for possible figures (Figure 3). An ANOVA showed this main effect to be significant $\left[F(4,140)=6.71, M S_{\mathrm{e}}=72,773\right]$. Significant priming was observed for the $0^{\circ}$ condition $[t(35)=3.60$; $83 \%$ of the participants showed this effect, $z=4.00]$, but not for other orientation conditions (all $t s<1.44$ ).

Analyses of recognition performance (Figure 3) were conducted on studied objects only, in order to assess the effect of orientation shifts on successful recognition performance. ANOVAs showed significant effects both for accuracy $\left[F(3,105)=10.91, M S_{\mathrm{e}}=0.0236\right.$; error rates are shown in parentheses in Figure 3] and RT $[F(3,105)$ $\left.=16.25, M S_{\mathrm{e}}=111,214\right]$. In addition, a contrast test (weights: $0^{\circ}=-3,60^{\circ}=-1,120^{\circ}=+1,180^{\circ}=+3$ ) revealed a significant linear effect on RTs $[F(1,105)=$ 40.61].

Differences between the patterns of orientation effects in the object possibility and old/new recognition tasks were assessed with an ANOVA on RTs, including the two factors of task (object possibility or recognition) and test condition (leaving out unstudied items). The interaction of these factors was significant $[F(3,105)=4.45$, 


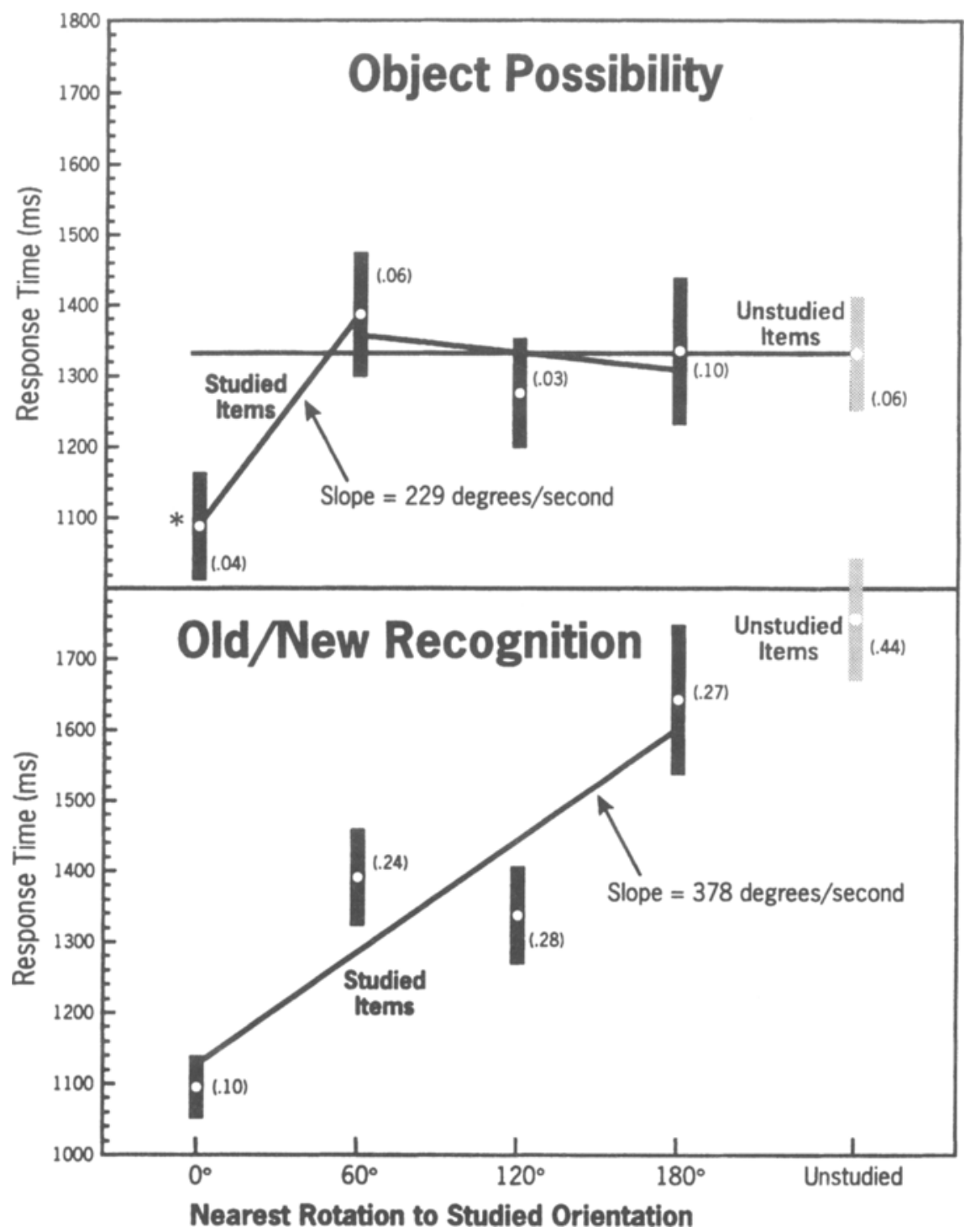

Figure 3. Object possibility and old/new recognition performance in Experiment 1. Rectangles represent standard errors of the mean, numbers in parentheses give error rates, and the asterisk denotes a priming effect significant by both $t$ and sign tests. Lines are least-square regression lines.

$\left.M S_{\mathrm{e}}=83,342\right]$, indicating that study-to-test orientation shifts had different effects on possibility priming and recognition memory. This result supports Cooper et al.'s (1991) conclusion that object possibility priming is eliminated by substantial study-test orientation shifts. That is, significant priming was observed in the form of reduced RTs when test figures were shown in the same orientation as they were studied, but significant priming was not observed when test figures were rotated $60^{\circ}$ or more from their studied orientations. This extreme form of viewpoint dependence has not been found in experiments employing traditional object recognition tasks, such as naming and old/new recognition. Indeed, the old/new recognition test in the present experiment re- vealed a standard viewpoint-dependent pattern of effects: Recognition performance was best when test objects were unrotated from studied viewpoints and gradually declined with increasingly large orientation shifts. This dissociation of possibility priming and recognition memory implies that the memory effects demonstrated on the object possibility task do not reflect the operation of everyday object identification processes.

This conclusion should be completely unsurprising, since the object possibility task does not require objects to be identified. Nevertheless, it is conceivable that the same encoded information could underlie both identification and priming but that the information could be used in different ways while the two tasks are being performed. 
When objects are viewed in the same orientation as they were studied, orientation-specific object representations might be accessed incidentally in the process of making possibility judgments and intentionally in the process of making identification judgments. When test objects are rotated, the intentional access involved in identification must still take place, since participants must access the representations if they are to successfully recognize the objects. However, during object possibility performance, it is possible that contact is made with encoded representations only if the representations are highly similar to test objects, and this condition may be violated when test objects are rotated $60^{\circ}$ or more from studied orientations.

If this hypothesis is correct, then smaller orientation changes might produce the same type of graded RT function found in old/new recognition and naming tasks. This prediction was tested in Experiment 2 using finer grained orientation shifts of $0^{\circ}, 15^{\circ}, 30^{\circ}, 45^{\circ}$, or $60^{\circ}$ between study and test.

\section{EXPERIMENT 2}

\section{Method}

Participants. Data were analyzed for 35 students from Oberlin College, Case Western Reserve University, and Brown University, all of whom participated in exchange for a \$6 payment.

Materials. Four additional objects were added to the 36 objects used in Experiment 1. The orientation at which each object was displayed in the experiments reported by Williams and Tarr (1997) was arbitrarily assigned as the $0^{\circ}$ orientation for the present experiment. Four copies of each object were then rotated $15^{\circ}, 30^{\circ}, 45^{\circ}$, or $60^{\circ}$ in the picture plane $(+=$ clockwise rotations; $-=$ counterclockwise). Since there was no reason to suspect different patterns of effects for rotations in either direction, each object was arbitrarily assigned to one of six sets: Set $A\left(+15^{\circ},+30^{\circ},-45^{\circ},-60^{\circ}\right)$, Set B $\left(+15^{\circ},-30^{\circ}\right.$, $\left.+45^{\circ},-60^{\circ}\right)$, Set $C\left(+15^{\circ},-30^{\circ},-45^{\circ},+60^{\circ}\right)$, Set D $\left(-15^{\circ},+30^{\circ}\right.$, $\left.+45^{\circ},-60^{\circ}\right)$, Set $\mathrm{E}\left(-15^{\circ},+30^{\circ},-45^{\circ},+60^{\circ}\right)$, and Set F $\left(-15^{\circ}\right.$,

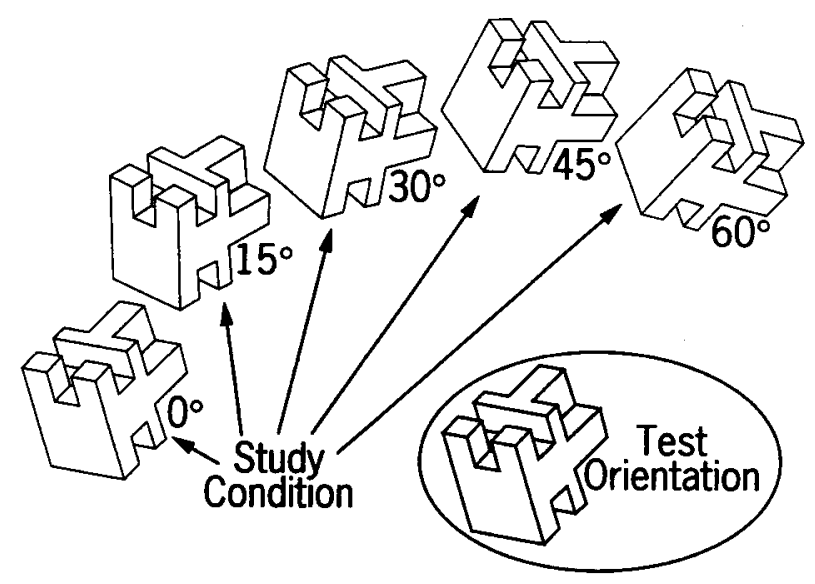

Figure 4. Stimulus design for Experiment 2. Objects were always tested in an arbitrarily chosen $0^{\circ}$ orientation, but they were studied in an orientation that was identical to the test orientation or was rotated by $\pm 15^{\circ}, \pm 30^{\circ}, \pm 45^{\circ}$, or $\pm 60^{\circ}$ in the picture plane. $\left.-30^{\circ},+45^{\circ},+60^{\circ}\right)$. Therefore, across objects, clockwise and counterclockwise rotations of each magnitude were seen equally often.

As illustrated in Figure 4, stimuli were studied in one of the $0^{\circ}$, $\pm 15^{\circ}, \pm 30^{\circ}, \pm 45^{\circ}$, or $\pm 60^{\circ}$ orientations and were always tested in the $0^{\circ}$ orientation. This design is the reverse of that used in Experiment 1 , where objects were always studied in the $0^{\circ}$ view and tested in rotated views. The present design afforded the advantage that unstudied objects were always tested in the same orientation, so no averaging across different unstudied orientations was necessary (although note that possibility performance for unstudied objects did not vary by viewpoint in Experiment 1).

Procedure. The procedure was identical to the one used in Experiment 1 , with the following exceptions. During the study phase, the participants performed the same tasks as before, but they saw figures for $3 \mathrm{sec}$ in the orientation, categorization, and pleasantness tasks and for $5 \mathrm{sec}$ in the surface-counting task (the participants seemed to become bored and therefore less motivated by the long exposure times in Experiment 1). Thirty objects were studied (vs. 24 in Experiment 1), 6 each in $0^{\circ}, 15^{\circ}, 30^{\circ}, 45^{\circ}$, and $60^{\circ}$ orientations. During the object possibility task, the participants had 80 critical test trials ( 30 studied and 10 unstudied objects, each in a possible and an impossible version) as well as the same 8 filler trials from Experiment 1 . All test items were shown in the $0^{\circ}$ orientation. During the old/new recognition task, the participants saw the 40 possible objects from the object possibility task.

Design. Independent variables of interest were test version (possible or impossible) and study condition $\left(0^{\circ}, 15^{\circ}, 30^{\circ}, 45^{\circ}, 60^{\circ}\right.$, or unstudied), both of which were varied within participants. An elaborate counterbalancing scheme was again required. Here, there were seven stimulus sets constructed such that, across sets, each object was represented once in 5 of the 6 study conditions and twice in the other condition (although not quite ideal, this design allowed us to test 10 unstudied objects and 6 objects in each of the other 5 study conditions while keeping the number of stimulus sets to a minimum). Analyses included data from 5 participants in each stimulus set condition.

\section{Results}

As in Experiment 1, there were no effects of study condition on either RTs or accuracy rates for impossible test figures (mean RTs for impossible objects were $1,252,1,279,1,321,1,302,1,236$, and $1,316 \mathrm{msec}$ for $0^{\circ}$, $15^{\circ}, 30^{\circ}, 45^{\circ}, 60^{\circ}$, and unstudied conditions, respectively), nor were there study condition effects on accuracy rates for possible test figures (all $F \mathrm{~s}<1.10$ ). However, RTs for possible test figures (Figure 5) did vary significantly by study condition $\left[F(5,170)=6.43, M S_{\mathrm{e}}=\right.$ $37,860]$. A linear contrast test on the means for studiedobject conditions only was significant $[F(1,136)=14.54$, $\left.M S_{\mathrm{e}}=41,054\right]$ and left very little residual variance $[F(3,174)=0.186]$.

Analyses on old/new recognition data (Figure 5) were again performed on the results of the five studied conditions, leaving out the unstudied condition. ANOVAs showed significant effects for both accuracy $[F(4,136)=$ $\left.5.27, M S_{\mathrm{e}}=0.0243\right]$ and RTs $\left[F(4,136)=6.47, M S_{\mathrm{e}}=\right.$ $59,607]$. A linear contrast on the participants' mean RTs (same weights as in object possibility analysis) was also significant $\left[F(1,136)=21.18, M S_{\mathrm{e}}=59,607\right]$.

In contrast to Experiment 1, patterns of effects on the object possibility and old/new recognition tasks in Exper- 


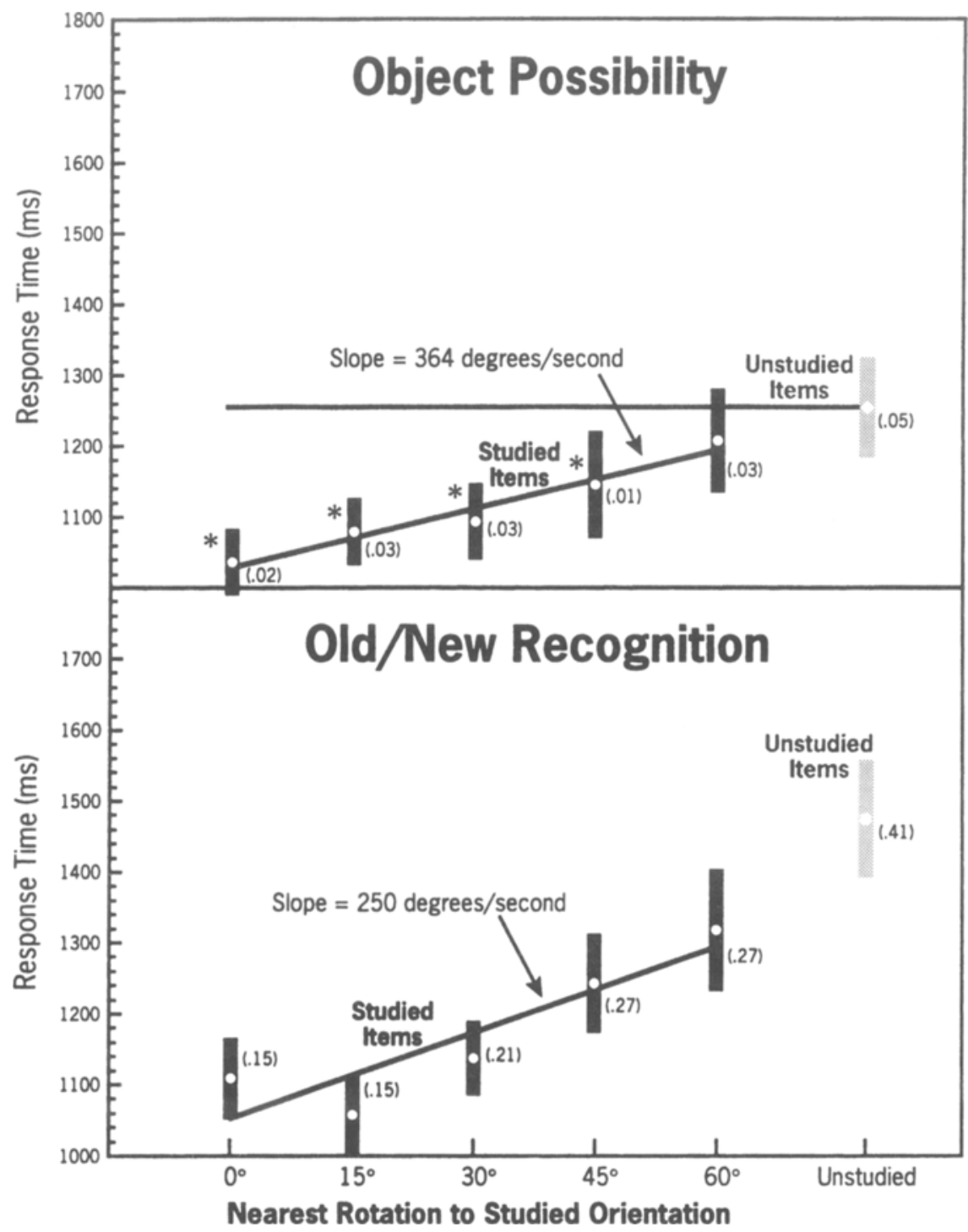

Figure 5. Object possibility and old/new recognition performance in Experiment 2. Rectangles represent standard errors of the mean, numbers in parentheses give error rates, and asterisks denote priming effects significant by both $t$ and sign tests. Lines are least-square regression lines.

iment 2 were very similar. Consistent with this conclusion, an ANOVA including the factors of task and study condition (excluding unstudied items) revealed no significant interaction $(F<1)$.

\section{Discussion}

The results of Experiment 2 show that object possibility priming and object identification suffer similar costs when objects are rotated $0^{\circ}-60^{\circ}$ in the picture plane between study and test: Performance on both the object possibility task and the old/new recognition task gradually declined throughout this range of orientation shifts. Furthermore, the slope of the functions relating RT to degree of orientation shift was remarkably similar for the two tasks (Figure 5), and these slopes were also similar to those found in Experiment 1 for object possibility performance between $0^{\circ}$ and $60^{\circ}$ orientation shifts and for old /new recognition performance over the entire range of $0^{\circ}-180^{\circ}$ shifts (Figure 3 ).

This correspondence in task performance between old/ new recognition and object possibility priming might seem at first contradictory to the many dissociations of the two tasks demonstrated by Schacter, Cooper, and their colleagues. For example, Schacter et al. (1990) demonstrated that old/new recognition is better following a "semantic" encoding task than following a "structural" task, whereas the magnitude of possibility priming was similar following both tasks. Nevertheless, access to seman- 
tic aspects of an object in an old/new recognition task is dependent on a participant's ability to first determine the visual identity of the object. Evaluation of visual identity is presumably based in part on the derivation of the object's structure, which is also the process assumed to be involved in object possibility priming (Cooper \& Schacter, 1992; Williams \& Tarr, 1997). Therefore, we should expect both dissociations and associations between old/ new recognition and object possibility performance, as demonstrated in Experiments 1 and 2.

Overall, then, the results of Experiment 2 are consistent with the two-part hypothesis put forward in the discussion following Experiment 1. First, both possibility priming and identification performance are mediated by orientation-specific object representations. Second, incidental access of these representations during the object possibility task occurs only when test figures are highly similar to studied figures. Apparently, greater-than- $60^{\circ}$ orientation shifts decrease the study-test similarity to the point where incidental access to encoded representations does not occur at all. The similarity in slopes between the two tasks leads us to add a third part to this hypothesis: The mechanisms leading to costs with orientation shifts are the same in the two tasks. In other words, the same process is being used to match perceived images to encoded representations in the object possibility and old/ new recognition tasks, so the mechanism incurs a similar cost due to shifts in the picture-plane orientation of objects.

What might this mechanism be? One popular explanation for rotation effects on identification performance has been that participants "mentally rotate" (Shepard \& Cooper, 1982) test objects into alignment with encoded representations (Jolicoeur, 1985; Tarr \& Pinker, 1989; Ullman, 1989). While these types of alignment processes could account for the costs associated with old/new recognition of rotated objects in the present experiments, the idea that intentional alignment underlies rotation costs on possibility priming is less appealing. Alignment would be useful when an encoded representation must be accessed, as in recognition tasks; but, in the object possibility task, participants are asked to make decisions solely on the test stimuli. Since possible and impossible figures were matched (Figure 1), explicit retrieval of ob-
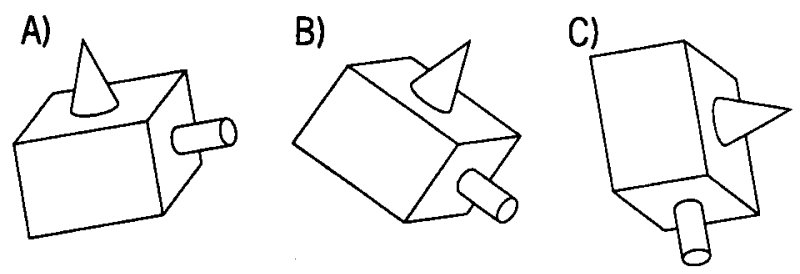

Figure 6. Three picture-plane rotated views of a simple 3-D object described in the text. Different structural descriptions may be derived from views $A$ and $C$, but the description derived from view B should match one or the other, given the qualitative coding of spatial relations in models such as Hummel and Biederman's (1992). ject representations would help very little in making possibility decisions (i.e., the trial might include an impossible figure that is very similar to a studied possible figure), so it is hard to see why an explicit alignment process would be performed. Furthermore, there is no a priori reason why alignment effects on priming would abruptly stop after $60^{\circ}$.

In a second class of models, objects are represented as viewpoint-invariant structural descriptions - propositionlike collections of primitive features that describe objects equally well from any (or at least many) different viewpoints (Biederman, 1987; Marr \& Nishihara, 1978). For example, the object in Figure 6A would be roughly described in Hummel and Biederman's (1992) implementation of Biederman's (1987) model as [brick-BELOWcone-BESIDE-cylinder]. A structural-description model could be adapted to perform object possibility decisions by responding "possible" if a valid structural description is computable from a test image or "impossible" otherwise. Schacter and Cooper (e.g., Cooper \& Schacter, 1992) have in fact proposed that a structural-description system of some sort underlies object possibility priming, although they have not explicitly outlined a mechanism for how structural descriptions contribute to possibility decisions.

Since the same description is generated when the object is seen from many different viewpoints, these models generally predict no effect of study-to-test orientation shifts, at least for rotations in depth. However, some predict viewpoint-dependent performance for picture-plane rotations, because such transformations can result in different structural descriptions being extracted for test objects than were extracted for studied objects (i.e., Figure $6 \mathrm{C}$ would be described as [brick-BESIDE-cone-ABOVEcylinder]). A related explanation was offered by Cooper et al. (1991) to explain their finding that $120^{\circ}$ and $180^{\circ}$ rotations completely disrupted possibility priming. For any one object, the effect of a picture-plane rotation on possibility priming should be discrete in these modelseither the study and test descriptions will match, in which case priming will be demonstrated, or they will mismatch, in which case no priming should be observed. Therefore, the fact that the orientation function was quite linear in Experiment 2 (Figure 5) tends to argue against this explanation. However, the rotation angle that causes a qualitatively different representation to be extracted could vary from object to object; so when many objects are employed and their results are averaged together, as in the present study, a structural-description theory can accommodate a linear response pattern over orientation-change conditions (Biederman \& Gerhardstein, 1993; Hummel \& Biederman, 1992).

A third class of object recognition models provides a more elegant explanation of orientation effects. These theories posit that perceived objects are compared directly (rather than following transformations as in alignment models) with object representations that are viewercentered-specified from the reference frame of the viewer, not the object (Bricolo, Poggio, \& Logothetis, 
1997; Edelman \& Weinshall, 1991; Perrett, Oram, \& Wachsmuth, 1998; Poggio \& Edelman, 1990). Since images of the same object from different vantage points will lead to different viewer-centered representations, these models posit that recognition decisions depend on computing the similarity between encoded and perceived views. That is, a perceived representation is compared with all (or at least many) encoded representations, and the best match determines what recognition decision is made. Since the match between perceived objects and encoded representations in the same orientation will always be greater than the match between misoriented objects and representations, viewer-centered similarity models predict that identification performance will degrade as the degree of misorientation increases.

Furthermore, object representations in these models are coded implicitly by altering units in a neural network that processes perceived images (neural-network processing allows similarity computations to be performed in parallel on a large number of encoded representations). Therefore, if the same neural network is used in the process of making possibility decisions, priming effects should automatically arise in the model: Because the network has been adapted to more efficiently process studied objects, decisions will be made more quickly about these objects than about unstudied objects. While the maximal benefit will be gained when test objects are unrotated, relative to studied objects, the partial match with slightly misoriented test objects should lead to partial priming effects. The results of Experiments 1 and 2 indicate that when the test objects used in the present study are rotated $60^{\circ}$ or more, studied objects are processed no more efficiently than unstudied objects in the object possibility test.

\section{EXPERIMENT 3}

Bülthoff and Edelman (1992) introduced a viewpoint manipulation that holds promise for distinguishing alignment, viewpoint-invariant structural-description, and viewer-centered similarity models. These researchers had participants study two different views of an object that were separated by a $75^{\circ}$ rotation. Following study, the object had to be recognized from studied viewpoints, from interpolated viewpoints that fell between the two studied views, and from extrapolated viewpoints that were outside the studied views. Experiment 3 invoked a similar design: Objects were studied in two versions that differed by a $90^{\circ}$ rotation in the picture plane and were then tested in a studied, an interpolated, or an extrapolated orientation, as illustrated in Figure 7.

The three classes of models presented above make different predictions for performance on the old/new recognition task. Interpolated views are similar to both studied views (differing by $45^{\circ}$ from each), whereas extrapolated views are highly similar to only one studied view (differ-

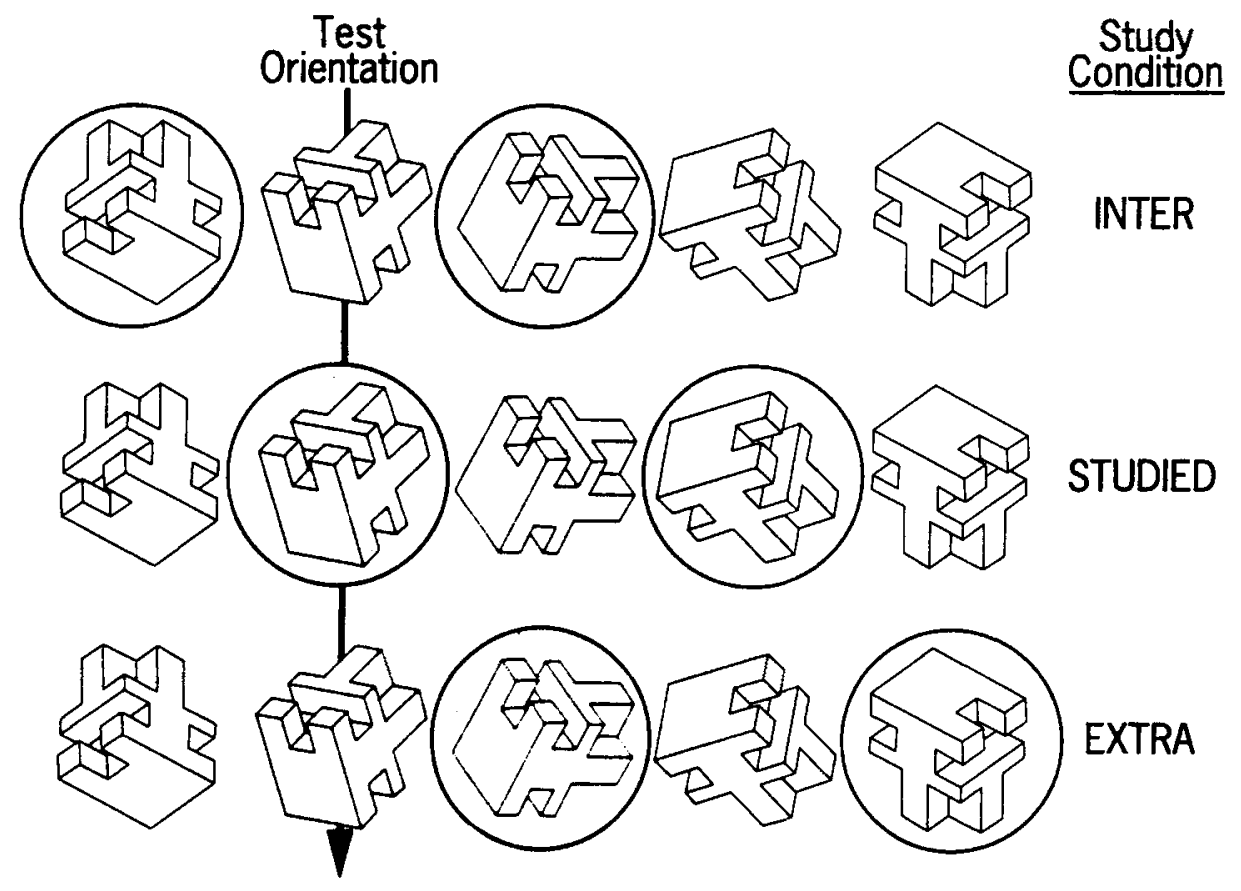

Figure 7. Stimulus design for Experiment 3. Objects were studied in two orientations that differed by $90^{\circ}$ and then were tested on one of the studied orientations (studied condition), an orientation that was between the two studied orientations (interpolation condition, INTER), or an orientation that was outside the two studied orientations (extrapolation condition, EXTRA). 
ing by $45^{\circ}$ from one view but $135^{\circ}$ from the other). Viewercentered similarity models (e.g., Poggio \& Edelman, 1990) therefore predict that interpolated views should produce more neural activity and thus be more efficiently recognized than extrapolated views, although recognition should be best for studied views. Alignment processes (e.g., Ullman, 1989) should operate no differently when dealing with interpolated and extrapolated views, so these models predict equivalent performance costs in the two conditions. Finally, in viewpoint-invariant structuraldescription models (Biederman, 1987), the description derived from an interpolated test view should be identical to the description derived from at least one of the two studied views (e.g., Figure $6 \mathrm{~B}, \mathrm{a}^{\circ}$ rotation of views $\mathrm{A}$ and $C$, would probably result in the same structural description as view A). This type of model thus predicts identical recognition performance from studied and interpolated views but slightly impaired performance from extrapolated views (since the latter will sometimes result in a structural description different from that derived from either of the studied views). Bülthoff and Edelman (1992), using rotations of objects in depth, found evidence supporting viewer-centered similarity models, in that interpolated views were recognized more accurately than extrapolated views, but not as accurately as studied views. We expected to replicate this finding, at least for the old/ new recognition task.

\section{Method}

Participants. Data were analyzed for 36 students from Yale and Brown Universities who participated for course credit or in exchange for a $\$ 6$ payment ( 3 of these participants were replacements for others who failed to follow task instructions).

Materials. The 36 objects used in Experiment 1 were used here. Objects were always tested in the same orientation as the test orientation from Experiment 2, and, also as in Experiment 2, study orientations varied from object to object. In the present experiment, objects were always studied in two orientations that differed by $90^{\circ}$. Figure 7 gives the relationship between studied and tested orientations. For objects in the studied condition, the test orientation was one of the studied orientations. For objects in the interpolation condition, the test orientation was interpolated between the two studied orientations, differing by $45^{\circ}$ from both. For objects in the extrapolation condition, the test orientation was extrapolated from the two studied orientations, differing by $45^{\circ}$ from one, but by $135^{\circ}$ from the other. For half of the objects, the extrapolated orientation was rotated clockwise from the nearest studied orientation, whereas, for the other half, the extrapolated orientation was rotated counterclockwise from the nearest studied orientation (since the interpolated orientation was between the two studied orientations, it was always rotated clockwise from one and counterclockwise from the other studied orientation).

Procedure. The procedure was identical to the one used in Experiment 1 , with the following exceptions. During the study phase, the participants performed only the orientation and categorization tasks (seeing each test stimulus for $3 \mathrm{sec}$ ) and saw each object twice in each task, once in each of the two studied orientations (Figure 7). Twenty-seven objects were studied, 9 each in the studied, interpolation, and extrapolation conditions. During the object possibility task, the participants had 72 critical test trials ( 27 studied and 9 unstudied objects, each in a possible and an impossible version) along with the 8 filler trials from Experiment 1 . All test items were shown in the same orientation as in Experiment 2. During the old/new recognition task, the participants saw the 36 possible objects from the object possibility task.

Design. Independent variables of interest were test version (possible or impossible) and study condition (studied, interpolation, extrapolation, or unstudied). Counterbalancing was more straightforward here than in Experiments 1 and 2, with four stimulus sets in which objects rotated among the four study conditions. Analyses included data from 9 participants in each stimulus set condition.

\section{Results}

As in the previous experiments, there were no effects of study condition on either RTs or accuracy rates for impossible object possibility test figures $[F(3,105)=1.10$, and $F<1$; mean RTs were $1,483,1,487,1,488$, and $1,388 \mathrm{msec}$ for the studied, interpolation, extrapolation, and unstudied conditions, respectively; none of these priming effects reached significance], nor were there study condition effects on accuracy rates for possible test figures on this test $[F(3,105)=1.49]$. However, RTs for possible test figures (Figure 8 ) varied significantly by study condition $\left[F(3,105)=3.42, M S_{\mathrm{e}}=39,287\right]$. As indicated in Figure 8, significant priming was observed for the studied and extrapolation conditions, but not for the interpolation condition.

Analyses on old/new recognition data (Figure 8) were again performed on the results of the three studied conditions, leaving out the unstudied condition. Omnibus ANOVAs were significant neither for accuracy $[F(2,70)=$ $\left.2.84, M S_{\mathrm{e}}=0.0196, p=.065\right]$ nor for RTs $[F(2,70)=$ $\left.1.66, M S_{\mathrm{e}}=49,159\right]$. On the basis of the results of Bülthoff and Edelman (1992), however, we expected the best performance on the studied condition, followed in turn by the interpolation and extrapolation conditions. We tested for this pattern with planned contrast tests (Rosenthal \& Rosnow, 1985, weights: studied $=-1$; interpolation $=$ 0 ; extrapolation $=+1$ ), which were significant for accuracy $\left[F(1,70)=5.67, M S_{\mathrm{e}}=0.0196\right]$, but not significant for RT $\left[F(1,70)=3.11, M S_{\mathrm{e}}=49,159, p=.082\right]$.

Although the ordering of the three studied-object conditions was different for the object possibility (studied $<$ extrapolation $<$ interpolation) and old/new recognition (studied < interpolation < extrapolation) tests, an ANOVA including the factors of task and study condition (excluding the unstudied condition) revealed no significant interaction $(F<1)$.

\section{Discussion}

Results of the old/new recognition task in Experiment 3 replicate those of Bülthoff and Edelman (1992): It was easiest for the participants to recognize objects presented in studied orientations, somewhat harder to recognize objects presented in orientations that fell between two studied orientations (the interpolation condition), and hardest to recognize objects in orientations outside the studied orientations (the extrapolation condition). This finding runs counter to alignment models (Ullman, 1989), 


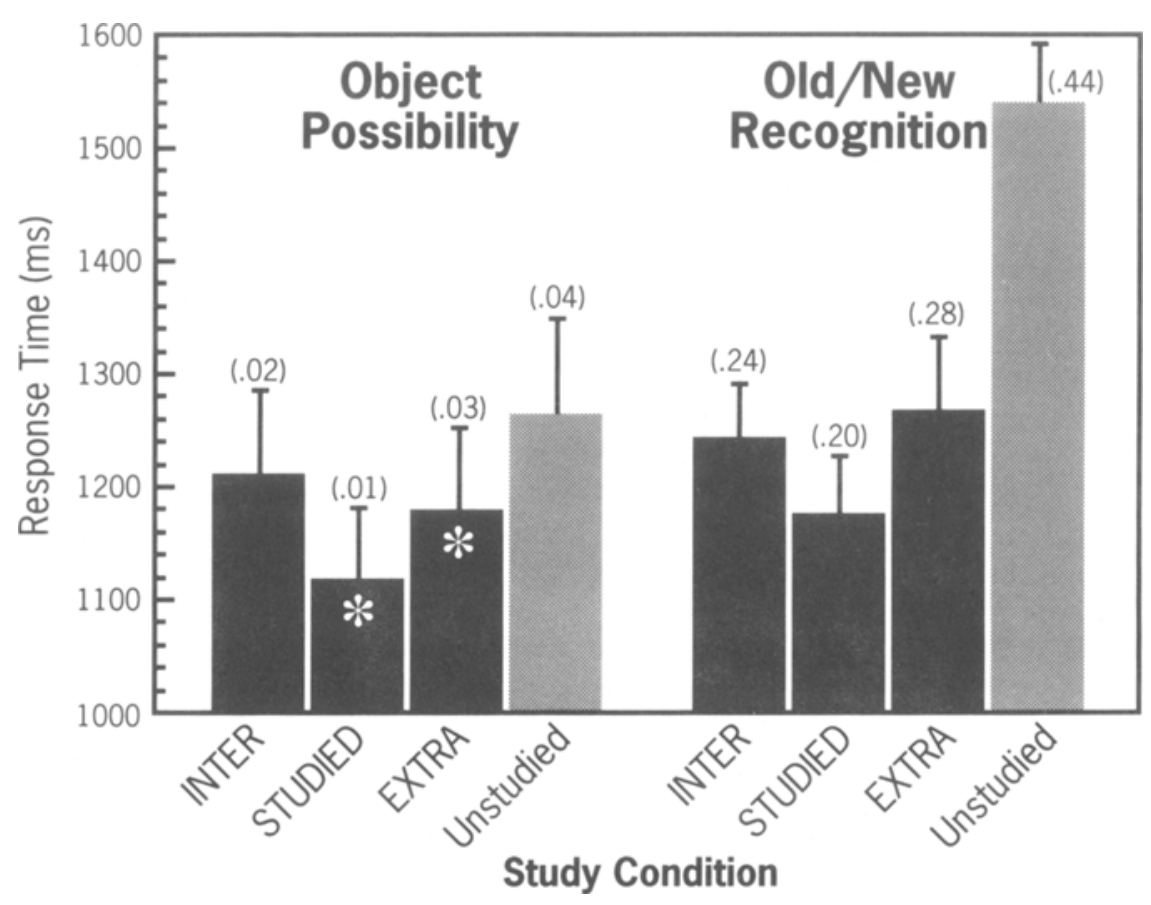

\begin{abstract}
Figure 8. Object possibility and old/new recognition performance in Experiment 3. Error bars indicate standard errors of the means, numbers in parentheses give error rates for each condition, and asterisks denote priming effects that were significant both by $t$ and sign tests.
\end{abstract}

which predict equivalent alignment times for interpolation and extrapolation conditions. Given the present experimental design, this finding is also incompatible with viewpoint-invariant structural-description models (Biederman, 1987), because since the two studied orientations differed by exactly $90^{\circ}$, one or the other of them should always have led to exactly the same structural description as the interpolation test orientation. Therefore, these models predict that performance in the interpolation condition should have been equivalent to performance in the studied condition, whereas the participants were both faster and more accurate on studied trials than on interpolation trials. ${ }^{1}$

In contrast, the pattern of effects on the old/new recognition task is nicely predicted by viewer-centered similarity models (Poggio \& Edelman, 1990). Test objects in the interpolated orientation are similar to two known views, whereas test objects in the extrapolated orientation are similar to only one of the known views. Therefore, the former objects should receive more activation and be recognized more quickly and accurately than the latter objects, as we observed.

Intriguingly, a slightly different pattern was found on the object possibility task. As was the case for old/new recognition, the participants were faster to make possibility decisions in the studied condition than in the interpolation condition ( 25 of the 36 participants showed this effect; $z=2.33$ ). Again, this finding argues against any model of object possibility priming invoking viewpointinvariant structural descriptions (i.e., Cooper et al., 1991), because one of the two studied orientations must have led to the same structural description as the interpolated test orientation. However, unlike old/new recognition performance, priming was not greater for interpolated orientations than for extrapolated orientations. In fact, the difference was in the opposite direction, but since the interaction between condition and task was not significant, we are not sure what to make of this finding at this time. In any case, the results of Experiment 3 reinforce those of Experiment 2 in that $45^{\circ}$ rotations of studied objects in the extrapolation condition were significantly primed on the object possibility test but were primed less than objects that were not rotated from studied orientations.

\section{GENERAL DISCUSSION}

The three experiments reported here reveal both correspondences and disparities between orientation effects on object possibility priming and old/new recognition performance. When test objects were rotated between $0^{\circ}$ and $60^{\circ}$ in the picture plane, relative to studied objects, both priming levels and recognition performance levels decreased at a rate of between $200^{\circ}-400^{\circ}$ /second. However, for study-test orientation shifts between $60^{\circ}$ and $180^{\circ}$, recognition performance continued to decline, whereas object possibility performance over this entire range was equivalent for studied and unstudied objects.

The close correspondence between object possibility priming and old/new recognition performance following $0^{\circ}-60^{\circ}$ orientation shifts suggests that both tasks draw on at least one common source of information. If we accept this suggestion, then a successful model of object 
recognition should account for orientation effects on both tasks. We have considered three types of object recognition models: alignment (Ullman, 1989), viewpointinvariant structural-description (Biederman, 1987), and viewer-centered similarity (Edelman \& Weinshall, 1991) models. An alignment account seems difficult (although not impossible) to reconcile with the implicit nature of memory retrieval revealed by object possibility priming. That is, alignment models are intuitively appealing when the task is to explicitly match a percept with a memory representation, as is the case with old/new recognition and object naming. ${ }^{2}$ In the object possibility task, however, participants are not asked to perform any such perceptmemory match, so it is hard to see why they would explicitly try to align studied and tested objects. Also, it is unclear why such a process would operate only on test orientations that are rotated $60^{\circ}$ or less from studied orientations.

In contrast to alignment models, the viewer-centered similarity models that have been proposed in the literature (Edelman \& Weinshall, 1991; Poggio \& Edelman, 1990 ) provide a natural account for priming effects, in that object representations are formed in these models by altering weights in neural networks designed to perform visual processing (Rouder, Ratcliff, \& McKoon, 1997). Since representations are stored directly in a processing system, priming effects will fall out of these models when previously studied test objects are processed differently than novel ones due to the altered weights. Furthermore, viewer-centered similarity models naturally predict orientation effects because rotated versions of studied objects will only partially match encoded representations of these objects (Bricolo et al., 1997; Perrett et al., 1998). That is, the extent to which processing of a studied object is facilitated is determined by the similarity between studied and tested images; as objects are rotated farther and farther from studied orientations, this similarity will decline, and the effect of study encounters on test performance (i.e., priming) will decline proportionally.
The difficulty for heretofore proposed viewer-centered similarity models (Bricolo et al., 1997; Edelman \& Weinshall, 1991; Poggio \& Edelman, 1990; Williams, 1997) in explaining the present results lies in the fact that they have not included any structural processing stages; thus, these implementations cannot account for how the object possibility task is even performed. However, the "pixelbased" processing in these models has not been intended as a strong claim about how objects are processed and represented in the human visual system. Rather, grayscale pixel values (Bricolo et al., 1997; Williams, 1997) and line junctions (Edelman \& Weinshall, 1991; Poggio \& Edelman, 1990) have been used because they are computationally convenient, allowing the development and testing of simulation models that test other fundamental assumptions, such as the utility of similarity-based processing in accounting for viewpoint effects.

The strengths and weaknesses of viewpoint-invariant structural description models are largely complementary. Because they posit representations that are viewpointinvariant and are compared to perceived objects in an allor-none fashion (as opposed to the graded matches computed in similarity-based models), structural-description models give less than satisfying accounts for viewpoint effects such as those found here. The results of Experiment 3 are especially problematic, since test objects in the interpolation condition should have matched at least one of the two structural descriptions for each studied object (Figure 6). On the other hand, structural descriptions of some sort would seem to be a requirement for performing the object possibility task, since impossible objects are, by definition, ones without valid structural descriptions.

A successful model for possibility priming, then, might include processing based on the similarity between perceived and encoded objects, along with representations that are viewer-centered, but specify objects' 3-D structures. No current model includes this combination of assumptions (and we will not attempt a full-blown model
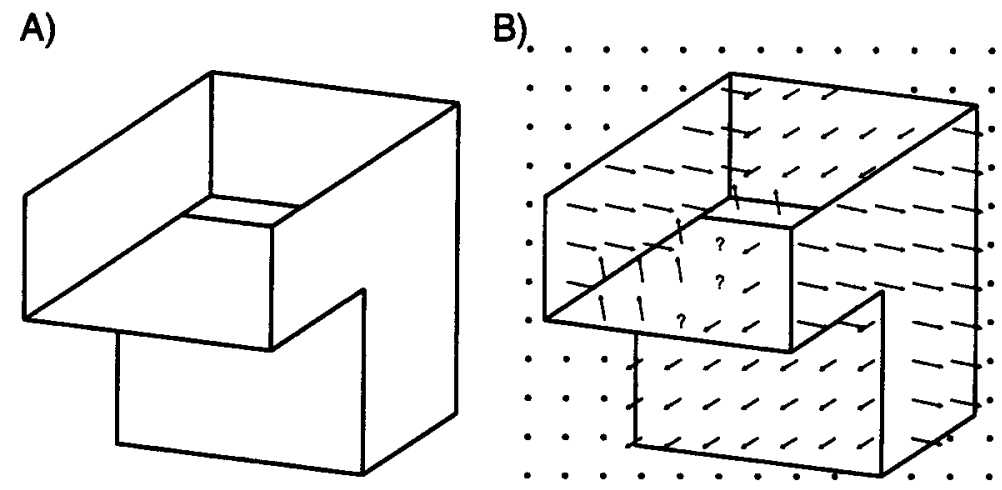

Figure 9. (A) A simple impossible object. The frontmost surface is primarily responsible for the object's impossibility, since it seems to be oriented toward the viewer on its right side but perpendicular to the ground plane on its left side. (B) $A$ representation of the 21/2-D sketch of this object (Marr, 1982), showing the slant and orientation of square-shaped patches of surface in the image. The patches marked with question marks indicate the area of structural ambiguity. 
here), but there is, in principle, no reason why such a model could not be built. Furthermore, as a starting point for the representational format of such an effort, we look (as have so many vision researchers in the past) to Marr (Marr, 1982; Marr \& Nishihara, 1978). The endpoint of Marr's vision of vision was a viewpoint-invariant structural description composed of generalized cylinders. However, Marr himself stated that his ideal of a 3-D, viewpoint-invariant representation had "yet to be put to empirical test" in 1982 (Marr, 1982, p. 327). On the basis of numerous findings of reliable viewpoint dependence in the recent literature (Bülthoff \& Edelman, 1992; Hayward, 1998; Perrett et al., 1998; Tarr, 1995; Tarr, Williams, Hayward, \& Gauthier, in press), it may be time to reject the viewpoint-invariant part of this ideal.

Although posited by Marr as a temporary buffer-like processing stage, his viewer-centered $2 \frac{1}{2} 2-D$ sketch could be closer to the type of representation used by the visual system to encode visual stimuli. The $21 / 2$-D sketch essentially specifies the surface discontinuities in an object along with the slant, tilt, and depth of each surface relative to the viewer (Marr, 1982, p. 277). This is exactly the type of information needed to determine that an impossible object cannot be instantiated in three dimensions. For example, the frontmost surface in Figure 9A seems to be oriented toward the viewer at its right edge but perpendicular to the ground plane at its left edge. Figure 9B is an attempt to depict the $2 \frac{1}{2} 2$-D sketch of this object. This representation makes explicit the fact that portions of the ambiguous surface face straight upward and straight frontward at the same time. If we assume a $2 \frac{1}{2}-\mathrm{D}$ visual processing stage, implemented as a neural network whose weights are changed as an image is processed, we can start to conceive of a mechanism by which object possibility priming can occur. We may further assume that the weights are altered only in response to unambiguous surfaces, since ambiguous surfaces in the real world are likely to reflect accidental views that are not representative of the true structure of an object. This assumption provides an explanation for Schacter et al.'s (1990) and Williams and Tarr's (1997) observations that priming is less strong for impossible objects than for possible objects.

By focusing, as we have here, on aspects of the visual input required to perform particular tasks and on specific ways in which these representations may cause priming, we are hopeful that future models will be able to provide more complete accounts for object possibility priming. Furthermore, by considering data from alternate tasks such as object possibility, object recognition researchers may discover a new source of information to constrain their own models and theories.

\section{REFERENCES}

BIEDERMAN, I. (1987). Recognition-by-components: A theory of human image understanding. Psychological Review, 94, 115-147.

Biederman, I., \& Gerhardstein, P. C. (1993). Recognizing depthrotated objects: Evidence and conditions for three-dimensional view- point invariance. Journal of Experimental Psychology: Human Perception \& Performance, 19, 1162-1182.

Bricolo, E., Poggio, T., \& Logothetis, N. K. (1997). 3D object recognition: A model of view-tuned neurons. In M. C. Mozer, M. I. Jordan, \& T. Petsche (Eds.), Advances in neural information processing systems 9 (pp. 41-47). Cambridge, MA: MIT Press.

Bülthoff, H. H., \& EdelmaN, S. (1992). Psychophysical support for a two-dimensional view interpolation theory of object recognition. Proceedings of the National Academy of Sciences, 89, 60-64.

COOPER, L. A., \& SCHACTER, D. L. (1992). Dissociations between structural and episodic representations of visual objects. Current Directions in Psychological Science, 1, 141-146.

Cooper, L. A., Schacter, D. L., \& Moore, C. (1991, November). Orientation affects both structural and episodic representations of threedimensional objects. Paper presented at the 32nd Annual Meeting of the Psychonomic Society, San Francisco.

Edelman, S., \& BülthofF, H. H. (1992). Orientation dependence in the recognition of familiar and novel views of three-dimensional objects. Vision Research, 32, 2385-2400.

EdElman, S., \& WEINSHALl, D. (1991). A self-organizing multiple-view representation of 3D objects. Biological Cybernetics, 64, 209-219.

HAYWARD, W. G. (1998). Effects of outline shape in object recognition. Journal of Experimental Psychology: Human Perception \& Performance, 24, 427-440.

Hummel, J. E., \& Biederman, I. (1992). Dynamic binding in a neural network for shape recognition. Psychological Review, 99, 480-517.

JOLICOEUR, P. (1985). The time to name disoriented natural objects. Memory \& Cognition, 13, 289-303.

MARR, D. (1982). Vision: A computational investigation into the human representation and processing of visual information. San Francisco: Freeman.

MARR, D., \& NishihaRA, H. K. (1978). Representation and recognition of the spatial organization of three-dimensional shapes. Proceedings of the Royal Society of London: Series B, 200, 269-294.

Perrett, D. I., Oram, M. W., \& Wachsmuth, E. (1998). Evidence accumulation in cell populations responsive to faces: An account of generalisation of recognition without mental transformations. Cognition, 67, 111-1145.

Poggio, T., \& Edelman, S. (1990). A network that learns to recognize three-dimensional objects. Nature, 343, 263-266

RATCLIFF, R., \& MCKoon, G. (1995). Bias in the priming of object decisions. Journal of Experimental Psychology: Learning, Memory, \& Cognition, 21, 754-767.

Rock, I., Di VITA, J., \& Barberto, R. (1981). The effect on form perception of change of orientation in the third dimension. Journal of Experimental Psychology: Human Perception \& Performance, 7, 719-732.

Rosenthal, R., \& Rosnow, R. L. (1985). Contrast analysis: Focused comparisons in the analysis of variance. Cambridge: Cambridge University Press.

Rouder, J. N., Ratcliff, R., \& McKoon, G. (1997, November). A model for implicit memory in object recognition. Paper presented at the 38th Annual Meeting of the Psychonomic Society, Philadelphia.

SCHACTER, D. L. (1992). Understanding implicit memory: A cognitive neuroscience approach. American Psychologist, 47, 559-569.

SChaCter, D. L., CoOper, L. A., \& Delaney, S. M. (1990). Implicit memory for unfamiliar objects depends on access to structural descriptions. Journal of Experimental Psychology: General, 119, 5-24.

Schacter, D. L., Reiman, E., Uecker, A., Polster, M. R., Sheng YUN, L., \& CoOPER, L. A. (1995). Brain regions associated with retrieval of structurally coherent visual information. Nature, 376, 587-590.

Shepard, R. N., \& CoOper, L. A. (1982). Mental images and their transformations. Cambridge, MA: MIT Press.

TARR, M. J. (1995). Rotating objects to recognize them: A case study on the role of viewpoint dependency in the recognition of threedimensional objects. Psychonomic Bulletin \& Review, 2, 55-82.

TARR, M. J., \& Pinker, S. (1989). Mental rotation and orientation dependence in shape recognition. Cognitive Psychology, 21, 233-282.

TARR, M. J., Williams, P., HaYWard, W. G., \& Gauthier, I. (in press). Object recognition is viewpoint-dependent. Nature Neuroscience. 
Ullman, S. (1989). Aligning pictorial descriptions: An approach to object recognition. Cognition, 32, 193-254.

Williams, P. (1995, November). Effects of picture-plane orientation, size, and color transformations on object decision priming. Paper presented at the 3rd Annual Workshop on Object Perception and Memory, Los Angeles.

Williams, P. (1997). Prototypes, exemplars, and object recognition. Unpublished doctoral dissertation, Yale University.

Williams, P., \& TARR, M. J. (1997). Structural processing and implicit memory for possible and impossible figures. Journal of Experimental Psychology: Learning, Memory, \& Cognition, 23, 1344-1361.

\section{NOTES}

1. Biederman and Gerhardstein (1993) claimed that old/new recognition is performed via mechanisms different from those used in "normal" object recognition and thus that results from the old/new recognition task cannot be used to test their structural-description model. On the other hand, Edelman and Bülthoff (1992) made the opposite claim, that old/new recognition-like tasks are "the closest to recognition in its everyday sense." As noted above, our view is that no one experimental task is perfectly diagnostic for normal, everyday object recognition performance; this view is what motivated us to compare old/new recognition and object possibility performance in the first place.

2. Note that object naming is an implicit memory task with respect to individual encounters with specific object exemplars, but the task requires explicit reference to generic memories of object classes (i.e., to say that an object is an elephant, one must directly match the perceived image to some kind of memory representation for previously encountered elephants). Object possibility decisions, on the other hand, depend neither on memories for study episodes nor on object-class representations. This potentially crucial distinction between the tasks is lost if both object possibility and object naming are simply classed as implicit memory tests.

(Manuscript received February 6, 1997; revision accepted for publication June 5, 1998.) 